\title{
Extramedullary Myeloma Spread Triggered by Surgical Procedures: An Emerging Entity?
}

\author{
Laura Rosiñol Carlos Fernández de Larrea Joan Bladé \\ Amyloidosis and Myeloma Unit, Hematology Department, IDIBAPS, Barcelona Hospital Clinic, Barcelona, Spain
}

Multiple myeloma (MM) is characterized by a proliferation of malignant plasma cells with a strong dependence on the bone marrow microenvironment. However, in patients with MM, the plasma cell proliferation can escape the microenvironment influences and result in extramedullary soft-tissue plasmacytomas (EMPs) which can constitute the most prominent feature of the disease. In fact, the reported incidence of EMPs in MM ranges from 7 to $18 \%$ at diagnosis and is as much as $20 \%$ at the time of relapse [1-4]. Soft-tissue plasmacytomas have two main origins: (1) direct growth from skeletal plasma cell tumors disrupting the cortical bone and (2) hematogenous spread [1]. It has been suggested that patients undergoing allogeneic transplantation, particularly those receiving reduced-intensity conditioning, may have a higher incidence of extramedullary disease (EMD) than those who receive an autologous stem-cell transplant. However, the incidence of EMD after allogeneic versus autologous stem-cell transplant has not been studied in comparable groups of patients. It has also been suggested that EMD is more frequent in patients exposed to novel agents. Nevertheless, a recent study showed that previous exposure to thalidomide or bortezomib was not associated with a higher risk of extramedullary relapse [3]. In addition, the study showed that up to $45 \%$ of patients with EMPs at diagnosis develop EMD at the time of relapse, and a multivariate regression analysis showed that the presence of EMD at diagnosis was the only predictor of extramedullary recurrence. The above features support the notion that characteristics inherent to the myeloma clone or host, rather than the treatment, are responsible for extramedullary spread in MM. As far as the development of EMD is concerned, local growth of soft-tissue masses arising from bone lesions is the most frequent mechanism, and second to this is the hematogenous spread that can involve any tissue or organ, the most frequent sites being the skin, liver, kidney, lymph nodes and central nervous system $[1,2]$. Positron emission tomography/computed tomography imaging may be very helpful in the search for EMD and these scans should be performed on all patients with suspicious of extramedullary involvement.

The mechanisms of extramedullary myeloma spread are poorly understood. Possibilities are: (1) decreased expression of adhesion molecules, particularly VLA-4 and CD44 as well as the loss of CD56, which can facilitate disease dissemination by impairing the adherence of myeloma cells to the bone marrow endothelium, (2)

\section{KARGER}

E-Mail karger@karger.com

www.karger.com/aha
(C) 2014 S. Karger AG, Basel

0001-5792/14/1321-0036\$39.50/0
Joan Bladé, MD

Servei d'Hematologia, Hospital Clínic de Barcelona

Villarroel 170

ES-08036 Barcelona (Spain)

E-Mail jblade@ clinic.ub.es 
low expression of chemokine receptors such as CCR1, CCR2 or downregulation of CXCR4 and its ligand SDF1 alpha, that are critically linked to the bone marrow homing of myeloma cells, (3) downregulation of tetraspanin expression, (4) increased angiogenesis or (5) bone marrow hypoxia [1]. In a recent study, it was reported that extramedullary involvement is more frequent with bone marrow plasma cells carrying the highrisk myeloma defined by gene expression profiling, including both the 70-gene and 80-gene University of Arkansas risk models [5].

In this issue of Acta Haematologica, Muchtar et al. [6] report a series of 3 patients with relapsed MM whose disease progressed with EMPs at the scars of previous surgical procedures. All 3 had initially been treated with novel agents and 2 of them with autologous stem-cell transplantation. All 3 failed to respond to different rescue therapeutic approaches after extramedullary progression at the scar site and had a short survival. Given the limited reports of EMPs arising from surgical scars, the authors suggest that myeloma relapse at the scar of a surgical procedure might go underrecognized or might indicate the existence of an emerging entity in the era of novel agents, particularly with the use of immunomodulatory drugs. Plasmacytomas triggered by surgical invasive procedures performed during the course of the disease can arise from laparotomy scars, catheter insertions or bone surgery. Interestingly, this presentation of extramedullary involvement can precede systemic relapses. Although the mechanisms of this type of relapse remain unclear, it has been speculated that the inflammatory process associated with tissue injury can facilitate the migration of myeloma cells into the skin or muscles and constitute a reservoir of dormant viable myeloma cells able to proliferate leading to the initially localized relapse $[6,7]$. Consistent with this hypothesis, it has been shown that in the severe combined immunodeficiency human myeloma model, cells from patients with EMPs injected directly to the fetal bone graft are capable of proliferating and grow in contiguous soft tissues beyond the bone graft, consistent with the clinical behavior of myeloma cells in some patients [8]. Clinicians should be aware of the possibility of this type of relapse in order to plan the best treatment approach, although with the current available therapies, including the so-called lymphoma-like regimens, the prognosis remains poor [1, 6-8].

It was emphasized in a recent editorial comment on extramedullary MM: 'extraosseous relapse is extra "bad news" but why?' [9]. In fact, many unsolved questions remain concerning its clinical aspects. (1) Is it really more frequent in the era of novel agents? (2) Is there a different pattern of extramedullary spread in patients treated with novel drugs? (3) Is it more frequent after allogeneic than after autologous stem-cell transplantation? (4) Could the so-called 'lymphoma-like' regimens be more effective than the typical 'anti-myeloma' regimens? There are questions that are important for future research. (1) Which mechanisms are involved in the hematogenous myeloma spread? (2) Are these mechanisms different from those involved in local growth from bone lesions? (3) What are the mechanisms of myeloma cell survival and growth at extramedullary sites? (4) What is the molecular genetic status of the malignant clone including genes involved in drug sensitivity and resistance? (5) Could genomic studies identify patients with MM particularly prone to developing extramedullary dissemination? [10]. There is no doubt that advances in research will result in a better understanding of extramedullary myeloma dissemination and, importantly, in a more effective therapy.

\section{Acknowledgment}

This work was supported in part by by grants (FIS 12/01093 and $\mathrm{RD} / 12 / 0036 / 0046)$ from the Instituto de Salud Carlos III, Spain.

\section{Disclosure Statement}

L.R. received honoraria for educational lectures from JanssenCilag and Celgene. C.F.L. received honoraria for educational lectures from Janssen-Cilag, Celgene and Novartis. J.B. received honoraria for consultation, educational lectures and research grants from Celgene and Janssen-Cilag.

References

Acta Haematol 2014;132:36-38

DOI: $10.1159 / 000354833$
- Bladé J, Fenández de Larrea C, Rosiñol L, Cibeira MT, Jimenez R, Powles R: Soft-tissue plasmacytomas in multiple myeloma: incidence, mechanisms of extramedullary spread, and treatment approach. J Clin Oncol 2011; 29:3805-3812.

$\checkmark 2 \mathrm{Wu}$ P, Davies FE, Boyd K, Thomas K, Dines S, Saso RM, Potter MN, Ethell ME, Shaw BE, Morgan GJ: The impact of extramedullary disease at presentation in outcome of myeloma. Leuk Lymphoma 2009;50:230-235.

-3 Varettoni M, Corso A, Pica G, Mengiacavalli S, Pascuto C, Lazzarino M: Incidence, presenting features and outcome of extramedullary disease: a longitudinal study on 1,003 consecutive patients. Ann Oncol 2009;21: 325-330. 
4 Rosiñol L, Oriol A, Teruel AI, Hernández D, López-Jiménez J, de la Rubia J, Granell M, Besalduch J, Palomera L, González Y, Etxebeste MA, Díaz-Mediavilla J, Hernández MT, de Arriba F, Gutiérrez NC, Martín-Ramos ML, Cibeira MT, Mateos MV, Martínez J, Alegre A, Lahuerta JJ, San Miguel J, Bladé J, Programa para el Estudio y la Terapéutica de las Hemopatías Malignas/Grupo Español de Mieloma (PETHEMA/GEM) group: Superiority of bortezomib, thalidomide and dexamethasone (VTD) as induction pre-transplantation therapy in multiple myeloma: a randomized phase III PETHEMA/GEM study. Blood 2012;120:1589-1596.
5 Usmani SZ, Heuck C, Mitchell A, Szymonifka J, Nair B, Hoering A, Alsayed Y, Waheed S, Haider S, Restrepo A, Van Rhee F, Crowley J, Barlogie B: Extramedullary disease portends poor prognosis in multiple myeloma and is over-represented in high-risk disease even in the era of novel agents. Haematologica 2012; 97:1761-1767.

-6 Muchtar E, Raanani P, Yeshurun M, Shpilberg O, Magen-Nativ H: Myeloma in scar tissue - an underreported phenomenon or an emerging entity in the novel agents' era? A single center series. Acta Haematologica 2014;132:39-44.

7 Fernández de Larrea C, Rosiñol L, Cibeira MT, Rozman M, Rovira M, Bladé J: Extensive soft-tissue involvement by plasmablastic myeloma arising from displaced humeral fractures. Eur J Haematol 2010;85:448-451.
Huang SY, Tien HF, Su FH, Hsu SM: Nonirradiated NOD/SCID-human chimeric animal model for primary human multiple myeloma: a potential in vivo culture system. Am J Pathol 2004;164:747-756.

9 Moreau P, Polliack A: Extramedullary multiple myeloma: extraosseous relapse is extra 'bad news', but why? Leuk Lymphoma 2012; 54:1349-1350.

10 Bladé J, Fernández de Larrea C, Rosiñol L: Extramedullary involvement in multiple myeloma. Haematologica 2012;97:1618-1619. 